\title{
Megakaryocytic Clustering in Chronic Myeloid Leukemia: Can it be a Predictor of Clinical Outcome?
}

\author{
Zunairah Mughal ${ }^{1}$, Hira Babar ${ }^{2}$, Sobia Ashraf ${ }^{1}$, Ambareen Hamid ${ }^{1}$, Abbas Khokhar $^{3}$ and Samina Qamar $^{1}$ \\ ${ }^{1}$ Department of Pathology, King Edward Medical University, Lahore, Pakistan \\ ${ }^{2}$ Department of Haematology, King Edward Medical University, Lahore, Pakistan \\ ${ }^{3}$ Department of Oncology, King Edward Medical University, Lahore, Pakistan
}

\begin{abstract}
Objective: To compare clinical outcome in patients of chronic myeloid leukemia (CML) with and without megakaryocytic clustering.

Study Design: Cross-sectional comparative study.

Place and Duration of Study: Pathology Department and CML Clinic Oncology Department, King Edward Medical University from March 2018 to March 2019.

Methodology: Ninety-four patients diagnosed with chronic phase of CML were included. Complete record of complete blood count, splenomegaly, findings of bone marrow aspirate and trephine biopsy was noted. Bone marrow trephine biopsy was reviewed for megakaryocytic clustering. Sokal scoring was done; and follow-up data for clinical outcome, i.e complete hematological response (CHR) at 3 months and major molecular response (MMR) at 6 months and 1 year (as per Institute's protocol) was obtained. All the data were analysed using SPSS version 25.

Results: Megakaryocytic clustering was present in 57 (60.6\%) patients and absent in 37 (39.4\%). In patients with megakaryocytic clustering, CHR was absent in $12(21.1 \%)$, MMR at 6 months was absent in 21 (36.8\%) and MMR at 1 year was absent in 25 (43.9\%) patients. In patients without megakaryocytic clustering, absent CHR, MMR at 6 months and MMR at 1 year were seen in 1 $(2.7 \%), 2(5.4 \%)$ and $2(5.4 \% \mathrm{x})$, respectively. The correlation of megakaryocytic clustering and high sokal score was found to be statistically significant with a $p$-value $<0.001$.

Conclusion: Patients with megakaryocytic clustering have poor clinical outcome as indicated by their sokal score, absent CHR, MMR at 6 months and 1 year.
\end{abstract}

Key Words: Chronic myeloid leukaemia, Megakaryocytic clustering, Complete haematological response, Major molecular response, Cytogenetic response.

How to cite this article: Mughal Z, Babar H, Ashraf S, Hamid A, Khokhar A, Qamar S. Megakaryocytic Clustering in Chronic Myeloid Leukemia: Can it be a Predictor of Clinical Outcome?. J Coll Physicians Surg Pak 2021; 31(01):34-38.

\section{INTRODUCTION}

Chronic myeloid leukemia (CML) is characterised by $5^{\prime}$ promotor on chromosome 9 translocation to $3^{\prime}$ exon on chromosome 22, resulting in constitutive activation ofJAK-STAT pathway leading to uncontrolled proliferation of myeloid series. ${ }^{1}$ The disease is more common in males aging 55-60 years. The prominent clinical features of disease include fatigue, anorexia, weight loss, massive spleen. Complete blood counts show abnormally high total leucocyte count with presence of myeloid series cells in all stages of maturation. It is often accompanied by anemia and/or thrombocytopenia in blood. ${ }^{2}$

Correspondence to: Dr. Sobia Ashraf, Department of Pathology, King Edward Medical University, Lahore, Pakistan

E-mail: s.qayyum81@gmail.com

Received: September 04, 2020; Revised: November 12, 2020;

Accepted: December 30, 2020

DOI: https://doi.org/10.29271/jcpsp.2021.01.34
The disease is characterised by 3 phases: chronic, accelerated, and blast. Majority of the patients present in the chronic phase, around $10 \%$ present in the accelerated phase, and another $10 \%$ in the blast phase. ${ }^{3}$ Bone marrow trephine findings range from hypercellular marrow with minimal fibrosis to advance fibrosis, ${ }^{4}$ with or without megakaryocytic clustering. These findings help in deciding about appropriate choice of treatment.

Megakaryocytic clustering is defined as a cluster of 3-5 megakaryocytes in trephine biopsy. They are pleomorphic with topographic disorganisation. The clusters may be present in sheets. ${ }^{5}$

Several scores are used for staging of this disease. These include Hashford, EUTOS and sokal score.

Sokal score is widely used in patients for risk stratification at the time of presentation. It takes into account four clinical variables: age,size of spleen, percentage of blast cells, and platelet count. The hazard ratio (Sokal score) is calculated by entering data in the following equation: $11 \operatorname{Exp}[0.116$ (age-43.4)] +0.0345 $($ spleen size-7.51) $+0.188[($ platelets $/ 700) 2-0.563]+0.0887$ 
(blast \%-2.10). Based upon score, patients are divided in three groups: low risk group (sokal score <0.8), intermediate group (sokal score 0.8-1.2), and high risk gourp (sokal score $>1.2$ ). ${ }^{6}$

Patients are followed up for complete hematological response (CHR) and major molecular response (MMR). CHR is defined as total leucocyte count of less than $10 \times 10 * 9 / \mathrm{L}$, platelet count of $450 \times 109 / \mathrm{L}$, absence of immature cells (blasts, promyelocytes, or myelocytes) in the peripheral blood, and disappearance of all clinical signs and symptoms including splenomegaly. MMR is achieved when BCR-ABL fusion gene cannot be demonstrated in the cases. $^{7,8}$

Tyrosine kinase inhibitors (TKI's) are a novel approach in management of CML. ${ }^{9}$ There are three generations of $\mathrm{TKI}^{\prime} \mathrm{S}$, Imatinib is first line drug, given to patients in chronic phase. ${ }^{10}$ It is given in a dose of $600 \mathrm{mg}$. It cause complete hematological response in $89 \%$ patients and complete cytogenetic response in $10 \%$. Median survival rate is 7 months. ${ }^{11}$

According to WHO guidelines, megakaryocytic clustering and fibrosis are taken as presumptive evidence of accelerated phase in the absence of any other criteria of accelerated phase. ${ }^{12}$

The objective of this study was to determine frequency of megakaryocytic clustering in patients of CML in chronic phase at the time of presentation and comparison of response to treatment with Imatinib in patients with or without megakaryocytic clustering.

\section{METHODOLOGY}

It was a retrospective study conducted at Mayo Hospital, Lahore in collaboration with CML Clinic Mayo Hospital and Hematology Section of Pathology Department, King Edward Medical University, from March 2018 to March 2019. All the diagnosed patients of CML in chronic phase with positive BCR/ABL fusion gene were included in the study regardless of the gender and age, and who were taking treatment with first line drugs and were compliant with a regular follow-up. All those diagnosed patients of CML with some additional chromosomal mutation or with JAK2 positivity, were excluded from the study.

After obtaining permission from University Ethical Review Board, all the data regarding personal information, medical history, physical examination, and investigations of included patients were recorded. The complete blood count for hemoglobin levels, total leucocyte count, differential leucocytic count, and platelet count done at the time of presentation, were reviewed. Findings of abdominal ultrasound/CT abdomen, for the presence of splenomegaly and size of spleen as per patient's record were also recorded after keeping full confidentiality.

Bone marrow trephine biopsy stained with hematoxylin and eosin done for diagnosis of disease, were re-examined for the presence of megakaryocytic clustering by two independent observers to minimise inter-observer bias. Megakaryocytic clustering is said to be present, if three or more clusters of five or more megakaryocytes are seen in an area of $1 \mathrm{~cm}$.
Treatment and follow-up response of all the patients were retrieved. The initial therapy in all patients was tablet Imatinib $400 \mathrm{mg}$. After three months, the patients were followed up for CHR. As per institutional policy, assessmet for CHR is done by complete blood count, peripheral smear, and ultrasound/CT abdomen. Those who failed to achieve $\mathrm{CHR}$ were prescribed an increased dose of $800 \mathrm{mg}$ of Imatinib. At 6 months and one year, all the patients were assessed for major molecular response. Ideally, cytogenetic analysis is to be done at 6 months and molecular analysis at 1 year as per the guidelines; but due to economic constraints, only molecular analysis is done both at 6 months and 1 yearas perinstitutional policy.

The results were recorded and data were analysed using SPSS V 25. Continuous variable like age, $\mathrm{Hb}$, TLC, spleen size, were described in terms of minimum and maximum values with mean \pm S.D and categorical variables like gender, were presented as frequencies.

Chi-square test was applied to determine correlation among qualitative variables and a $p$-value of $<0.05$ was considered statisticallysignificant.

\section{RESULTS}

A total of 94 patients of CML in CP were identified during the defined study period of 6 months. There were 51 (54.3\%) male patients and $43(45.7 \%)$ female patients with a mean age of $47.02 \pm 9.3$ years. Other characteristic variables are shown in Tablel.

The bone marrow trephine biopsy slides of all the patients were evaluated for megakaryocytic clustering. It was present in 57 $(60.6 \%)$ of the patients. Bone Marrow trephine biopsy figures without megakaryocytic clustering and with megakaryocytic clustering are shown below (Fig $1 \mathrm{a}$ and $1 \mathrm{~b}$, respectively).

The sokal score of each patient was calculated according to the formula mentioned above. A total of 18 (19.1\%) patients had high sokal score, 33 (35.1\%) had intermediate sokal score and $43(45.7 \%)$ had low sokal score. The correlation of megakaryocytic clustering and high sokal score was found to be statistically significant $(p<0.001$, Table II).

The percentage of patients with megakaryocytic clustering and absent CHR, MMR at 6 months and 1 year is illutrated in Figure 2 .

All the patients werefollowed up to assess complete hematological response at the end of three months and MMR after 6 months and 1 year. The cytogenetic response is not done owing to institutional policy. It was observed that $\mathrm{CHR}$ was achieved in 81 (86.2\%) and absent in 13 (13.8\%) patients; out of which, 12 (92.3\%) patients had megakaryocytic clustering ( $p$-value = 0.012). Similarly, MMR at 6 months was absent in 23 (24.5\%) patients. Out of them, megakaryocytic clustering was observed in 21 (91.3\%, p-value = 0.001). At 12 months, MMR was absent in $27(28.7 \%)$ patients, out of which 25 (92.6\%) (p-value $<0.001$ ) had megakaryocytic clustering (Table II). 
Table I: Demographic and hematological variables of CML-CP patients.

\begin{tabular}{|c|c|c|c|}
\hline Variables & Mean \pm S.D. & Minimum & Maximum \\
\hline Age (years) & $47.02 \pm 9.3$ & 28 & 67 \\
\hline $\mathrm{Hb}(\mathrm{g} / \mathrm{dl})$ & $9.97 \pm 1.8$ & 5.5 & 13.1 \\
\hline TLC $\left(10^{3} u L\right)$ & $126.34 \pm 42.1$ & 34 & 212 \\
\hline Platelets ( $\left.10^{9} \mathrm{uL}\right)$ & $386.95 \pm 161.7$ & 112 & 890 \\
\hline Blast count (\%) & $3.80 \pm 4.48$ & 1 & 9 \\
\hline Spleen size $(\mathrm{cm})$ & $15.22 \pm 1.8$ & 9 & 23 \\
\hline
\end{tabular}

Table II: Relationship of megakaryocytic clustering with gender, sokal score, CHR, MMR at 6 months and 1 year.

\begin{tabular}{|c|c|c|c|c|c|c|c|c|c|c|c|c|}
\hline & & \multicolumn{2}{|c|}{ Gender } & \multicolumn{3}{|c|}{ Sokal } & \multicolumn{2}{|c|}{ CHR } & \multicolumn{2}{|c|}{ MMR at 6 months } & \multicolumn{2}{|c|}{ MMR at 12 months } \\
\hline & & $\begin{array}{l}\text { Female } \\
\mathbf{N}=43\end{array}$ & $\begin{array}{c}\text { Male } \\
\mathrm{N}=51\end{array}$ & $\begin{array}{c}\text { High } \\
N=18\end{array}$ & $\begin{array}{l}\text { Intermediate } \\
\quad \mathbf{N}=33\end{array}$ & $\begin{array}{c}\text { Low } \\
N=43\end{array}$ & $\begin{array}{c}\text { Absent } \\
\mathrm{N}=13\end{array}$ & $\begin{array}{c}\text { Present } \\
\mathbf{N}=\mathbf{8 1}\end{array}$ & $\begin{array}{c}\text { Absent } \\
\mathrm{N}=23\end{array}$ & $\begin{array}{c}\text { Present } \\
\mathrm{N}=71\end{array}$ & $\begin{array}{l}\text { Absent } \\
N=27\end{array}$ & $\begin{array}{l}\text { Present } \\
\mathrm{N}=67\end{array}$ \\
\hline \multirow{3}{*}{$\begin{array}{l}\text { Megakaryocytic } \\
\text { clusters }\end{array}$} & Absent & $19(44.2 \%)$ & $18(35.3 \%)$ & $1(5.6 \%)$ & $9(27.3 \%)$ & $27(62.8 \%)$ & $1(7.7 \%)$ & $36(44.4 \%)$ & $2(8.7 \%)$ & $35(49.3 \%)$ & $2(7.4 \%)$ & $35(52.2 \%)$ \\
\hline & Present & $24(55.8 \%)$ & $33(64.7 \%)$ & $17(94.4 \%)$ & $24(72.7 \%)$ & $16(37.2 \%)$ & $12(92.3 \%)$ & $45(55.6 \%)$ & $21(91.3 \%)$ & $36(50.7 \%)$ & $25(92.6 \%)$ & $32(47.8 \%)$ \\
\hline & $p$-values & \multicolumn{2}{|c|}{0.379} & \multicolumn{3}{|c|}{$<0.001$} & \multicolumn{2}{|c|}{0.012} & \multicolumn{2}{|c|}{0.001} & \multicolumn{2}{|c|}{$<0.001$} \\
\hline
\end{tabular}

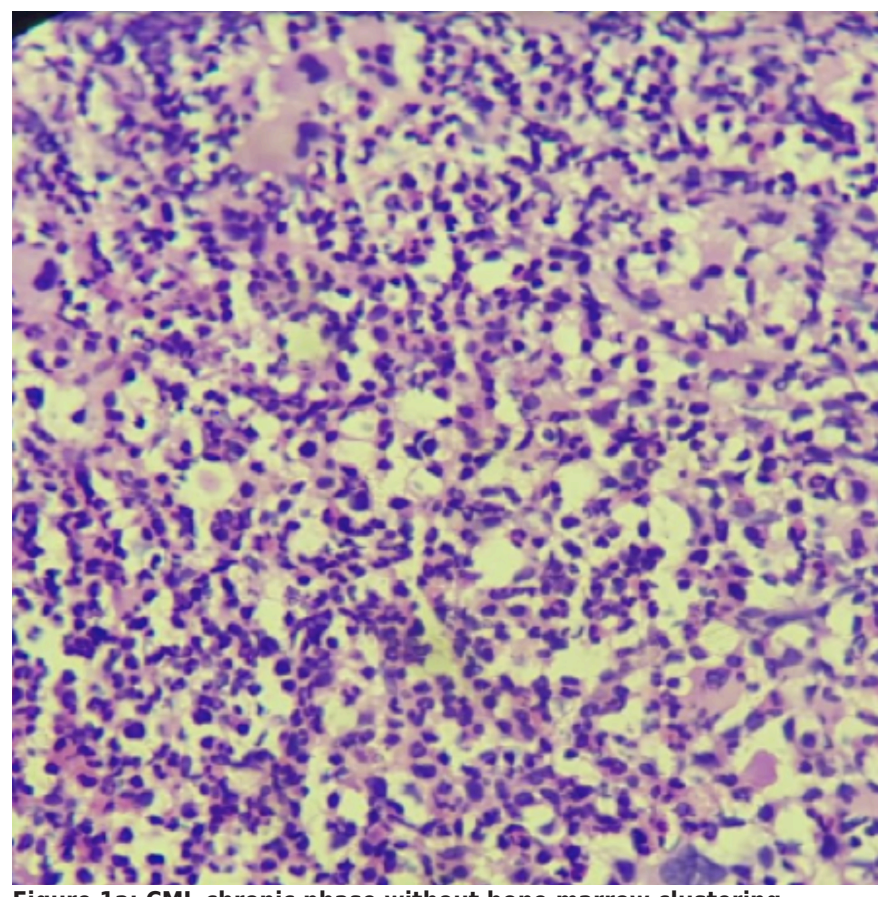

Figure 1a: CML chronic phase without bone marrow clustering.

\section{DISCUSSION}

CML involves malignant transformation of multipotent progenitor cells including granulocytes and megakaryocytes, erythroid cells and macrophages at a variable extent. In this study, 94 patients of CML in chronic phase were included. Mean age of the patient was $47.02 \pm 9.3$ years with male predominance. Average hemoglobin level was found to be 9.9 , and anemia was seen in 47 patients, mean platelet count was $386,000 / \mathrm{ul}$, and was found to be normal or slightly on the lower side. Mean WBC count was 126,000/ul. These findings are in accordance with study by Hamid et al. ${ }^{13}$

Average spleen size was $15 \mathrm{~cm}$ and splenomegaly was seen in 23 patients. In a study by Kumar, no significant splenomegaly was found among the patients and they also found platelet count to be slightly higher in the patients having CML. ${ }^{14}$

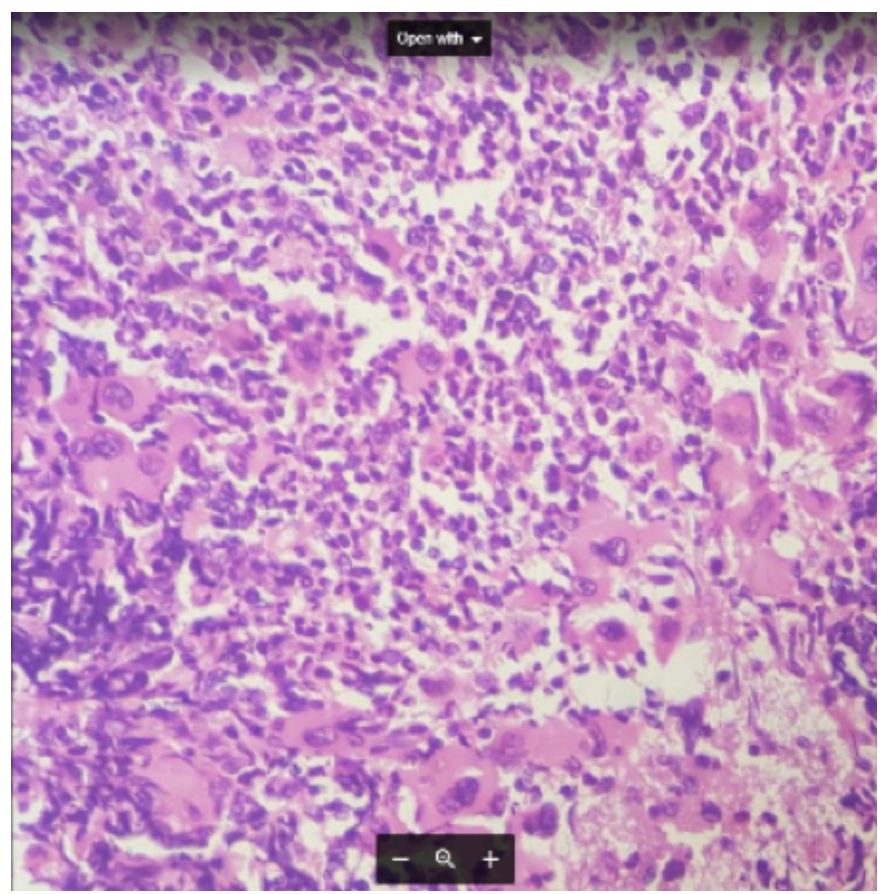

Figure 1b: CML chronic phase with bone marrow clustering.

Sokal Index for CML predicts survival based on clinical and laboratory investigation, done before the commencement of treatment. Sokal score was high in $18(19.1 \%)$ patients, intermediate in $33(35.1 \%)$, and low in $43(45.7 \%)$ patients. Similar results were found in the study conducted by Laskshmaiha in India. ${ }^{15}$

Megakaryocytes were the main focus of this study. The megakaryocytic number and clustering was closely observed in the trephine biopsy slides. The megakaryocytic number was raised in $57(60.6 \%)$ of cases observed. Megakaryocytic clustering was seen in all these patients; whereas, dwarf forms were seen in $20 \%$ of the cases. 


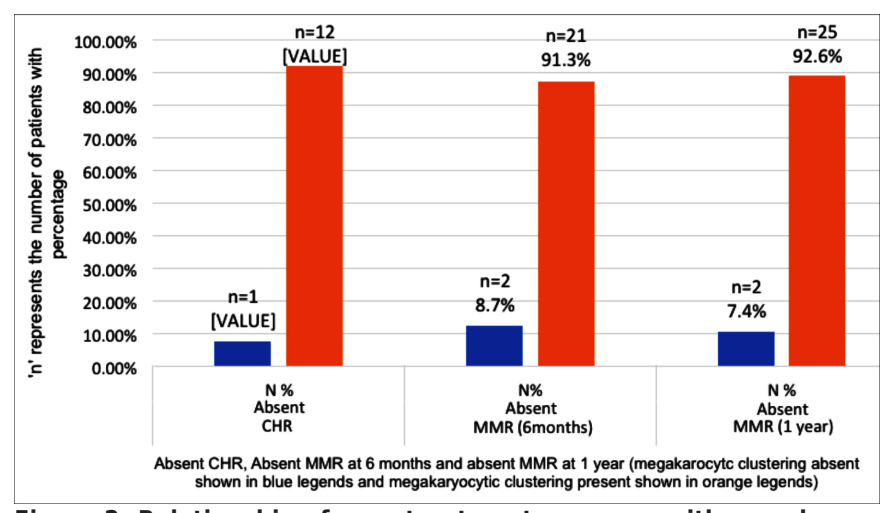

Figure 2: Relationship of poor treatment response with megakaryocytic clustering.

Arun et al. has studied the number and morphologic features of megakaryocytes in CML patients. The number was raised in $67 \%$ patients and $32 \%$ patients had megakaryocytic clustering. They divided patients according to classification of CML by Burkhardt into CML-GM (granulocytic megakaryocytic) and CML-G (granulocytic). Increased number and clustering was seen exclusively in CML-GM group. ${ }^{16}$

All patients were followed for at least 1 year and treatment response was noted. Good response in the form of complete CHR, MMR1 and MMR2 was seen in 57 patients and bad response in the form of failure of either one or all $C H R$, MMR 1 and MMR 2 was seen in 37 patients.

The cytogenetic response is not done owing to institutional criteria. However for the correlation of responses as shown by Jabbour et al. in updates of CML, instead of repeated bone marrow biopsy, a complete CCyR is equal to negative FISH test and BCR-ABL $<1 \%$ and partial CCyR is equal to BCR-ABL $<10 \% .{ }^{17}$ It was observed that CHR was achieved in $81(86.2 \%)$ and absent in $13(13.8 \%)$ patients, out of which $12(92.3 \%)$ patients has megakaryocytic clustering ( $p$ $=0.012$ ). In a study by Hagop Kartarijan, CHR was observed in $89 \%$ of the patients. ${ }^{18}$

Similarly, MMR at 6 and 12 months was absent in 23 (24.5\%) and $27(28.7 \%)$ patients in which megakaryocytic clustering was observed in $21(91.3 \%, p=0.001)$ and $25(96.2 \%, p$ $<0.001$ ) patients.

The correlation of sokal score with megakaryocytic clustering was done, which showed that nearly all the patients with high sokal score had megakaryocytic clustering seen in their trephine biopsy. Those with intermediate sokal score, $72 \%$ of those had clustering, showing a significant correlation. Similar results were seen in a study by Theil el al., in which increased CD 61 positive megakaryocytic cells were associated with increased tumor burden and advanced stage of disease. ${ }^{19}$

Similar correlation was seen in a study by Forrest et al., in which CHR and MMR correlated with the sokal score. ${ }^{20}$ Nachi et al. has conducted study on effect of gender on treatment outcome. In that study, females were found to have far better outcome (MMR 80 vs. 45\%). Similar results were found in our study as males had more megakaryocytic clustering than females. Bad response was seen in 33 males and 24 females and good response was seen in 18 males and 19 females. ${ }^{21}$

\section{CONCLUSION}

Patients with megakaryocytic clustering have poor clinical outcome as indicated by their sokal score, absent complete hematological response (CHR), molecular response at 3 months and 1 year. Trephine biopsy of all the patients with chronic CML should be assessed for megakaryocytic clustering, as it is presumptive of poor prognosis in $\mathrm{CML}$ patients.

\section{ETHICAL APPROVAL:}

Ethical approval of this study was obtained from King Edward Medical University Ethics Committee prior to initiation of the research work.

\section{PATIENTS' CONSENT:}

Informed consents were obtained from all patients to publish the data concerning this study.

\section{CONFLICT OF INTEREST:}

Authors declared no conflict of interest.

\section{AUTHORS' CONTRIBUTION:}

$\mathrm{ZM}, \mathrm{HB}$ : Data acquisition, analysis and drafting of the study, agreement to be accountablefor all aspects of the work in ensuring that questions related to the accuracy or integrity of any part of the work are appropriately investigated and resolved.

SA, AH, AK: Conception and design of the study, review and final approval of the draft, agreement to be accountable for all aspects of the work in ensuring that questions related to the accuracy or integrity of any part of the work are appropriately investigated and resolved.

SQ: Drafting of the study, data analysis and interpretation, agreement to be accountable for all aspects of the work in ensuring that questions related to the accuracy or integrity of any part of the work are appropriately investigated and resolved.

\section{REFERENCES}

1. Zhang B, Li L, Ho Y, Li M, Marcucci G, Tong W, et al. Heterogeneity of leukemia-initiating capacity of chronic myelogenous leukemia stem cells. J clinical Investigation 2016; 126(3):975-91. doi: 10.1172/JCI79196.

2. Kansal R. Acute myeloid leukemia. Tumors and cancers: Endocrine glands-blood-marrow-lymph. 2017.

3. Karkuzhali P, Shanthi V, Usha T. A case of chronic myeloid leukemia presenting as megakaryocytic blast crisis (AML M7). Ecancermedicalscience 2013; 7:375. doi: 10.3332/ecancer.2013.375. 
4. Kumar S, Gupta VK, Bharti A, Meena LP, Gupta V, Shukla J. A study to determine the clinical, hematological, cytogenetic, and molecular profile in CML patient in and around Eastern UP, India. J family Medicine Primary Care 2019; 8(7):2450. doi: 10.4103/jfmpc.jfmpc_307_19.

5. Khonglah Y, Basu D, Dutta TK. Bone marrow trephine biopsy findings in chronic myeloid leukemia. Malaysian J Pathology 2002; 24(1):37-43.

6. Usman M, Syed NN, Kakepoto GN, Adil SN, Khurshid M. Chronic phase chronic myeloid leukemia: response of Imatinib mesylate and significance of Sokal score, age and disease duration in predicting the hematological and cytogenetic response. JAPI 2007; 55:103-07.

7. Baccarani M, Pane F, Saglio G. Monitoring treatment of chronic myeloid leukemia. Haematologica 2008; 93(2): 161-9. doi: 10.3324/haematol.12588.

8. Saussele S, Hehlmann R, Fabarius A, Jeromin S, Proetel U, Rinaldetti $S$, et al. Defining therapy goals for major molecular remission in chronic myeloid leukemia: results of the randomized CML Study IV. Leukemia 2018; 32(5):1222-8.

9. Bhatia R. Novel approaches to therapy in CML. Hematology Am Soc Hematol Educ Program 2017; 2017(1):115-20. doi: 10.1182/asheducation-2017.1.115.

10. Goldman JM. Treatment strategies for CML. Best Practice \& Research Clinical Haematol 2009; 22(3):303-13.

11. Rosti G, Martinelli G, Bassi S, Amabile M, Trabacchi E, Giannini $B$, et al. Molecular response to imatinib in late chronic-phase chronic myeloid leukemia. Blood 2004; 103(6): 2284-90.

12. Shaffer LG. Editors. An International System for Human Cytogenetic Nomenclature. 2013.

13. Hamid A, Ashraf S, Qamar S, Naveed MA, Hameed A, Farooq MA. Myelofibrosis in patients of chronic myeloid leukemia in chronic phase at presentation. J Coll Phys Surg Pakistan 2019; 29(11):1096-100. doi: 10.29271/jcpsp.2019.11.1096.

14. Arunachalam AK, Jain M, Kumar A, Kushwaha R, Singh US,
Tripathi AK. Megakaryocytes in chronic phase of chronic myeloid leukemia: A descriptive case series. Annals Pathol Labor Med 2016; 3(3):A176-182.

15. Kuntegowdanahalli LC, Kanakasetty GB, Thanky AH, Dasappa L, Jacob LA, Mallekavu SB, et al. Prognostic and predictive implications of Sokal, Euro and EUTOS scores in chronic myeloid leukaemia in the imatinib era-experience from a tertiary oncology centre in Southern India. Ecancermedicalscience 2016; 10.

16. Burkhardt R, Bartl R, Jäger K, Frisch B, Kettner G, Mahl G, et al. Working classification of chronic myeloproliferative disorders based on histological, haematological, and clinical findings. J Clin Pathol 1986; 39(3):237-52. doi: 10.1136/jcp. 39.3.237.

17. Jabbour E, Kantarjian H. Chronic myeloid leukemia: 2018 update on diagnosis, therapy and monitoring. Am J Hematol 2018; 93(3):442-59. doi: 10.1002/ajh.25011.

18. Cortes J, Talpaz M, O'Brien S, Jones D, Luthra R, Shan J, et al. Molecular responses in patients with chronic myelogenous leukemia in chronic phase treated with imatinib mesylate. Clin Cancer Res 2005; 11(9):3425-32. doi: 10.1158/ 1078-0432.CCR-04-2139.

19. Thiele J, Kvasnicka HM, Fischer R, Diehl V. Clinicopathological impact of the interaction between megakaryocytes and myeloid stroma in chronic myeloproliferative disorders: a concise update. Leukemia \& lymphoma 1997; 24(5-6): 463-81. doi: 10.3109/10428199709055584.

20. Forrest DL, Trainor S, Brinkman RR, Barnett MJ, Hogge DE, Nevill TJ, et al. Cytogenetic and molecular responses to standard-dose imatinib in chronic myeloid leukemia are correlated with Sokal risk scores and duration of therapy but not trough imatinib plasma levels. Leukemia research 2009; 33(2):271-5. doi: 10.1016/j.leukres.2008.07.015.

21. Nachi M, Kihel I, Guella D, Dali-Ali A, Abed A, Boukhatmi Y. Sex and major molecular response to imatinib treatment for patients with chronic myeloid leukemia. Biochem Pharmacol (Los Angel) 2019; 8(263):2167-0501. doi: 10.4172/21670501.1000263. 